\title{
Special Volume devoted to the 15th International Frankia and Actinorhizal Plant Meeting
}

\author{
Eugenia Chaia $\cdot$ Jeffrey O. Dawson $\cdot$ Luis G. Wall
}

Published online: 1 January 2010

(C) Springer Science+Business Media B.V. 2009

The 15th International Frankia and Actinorhizal Plant Meeting was held from October 19th to 23rd, 2008, in Bariloche, Argentina. Frankia is an actinomycete that nodulates roots of a group of angiospermous plants. Frankia fixes atmospheric nitrogen symbiotically with its specific host plants in 24 genera within eight families.

This was the first of the biennial meetings to be held in South America and only the second, after New Zealand, to be held outside of North America and Europe. Thirty four scientists and students from nine different countries in South America, North America, Europe and Asia attended. The site of the meeting, Bariloche, is in the Patagonian region of the southern Andean Mountains, where a distinct group of actinorhizal plants and their Frankia symbionts occur. These plants are in the tribe Colletiae (family Rhamnaceae) and the meeting participants had the opportunity to explore their natural environs from steppe to

E. Chaia $(\bowtie)$

Universidad Nacional del Comahue,

Centro Regional Universitario Bariloche,

Quintral 1250,

Bariloche 8400, Argentina

e-mail: echaia@crub.uncoma.edu.ar

J. O. Dawson

Department of Natural Resources and Environmental Sciences,

University of Illinois,

Urbana, IL 61801, USA

e-mail: jdawson2@illinois.edu

\section{G. Wall}

Programa de Investigación en Interacciones Biológicas,

Departamento de Ciencia y Tecnología,

Universidad Nacional de Quilmes,

Roque Sáenz Peña 352,

Bernal 1876BXD, Argentina

e-mail: 1gwall@unq.edu.ar humid forest in the Nahuel Huapi National Park near Bariloche and to hear reports of the most recent research on this actinorhizal group.

Much research progress has been made on Frankia and on actinorhizal plants since the first meeting was held at Harvard Forest in Petersham, Massachusetts in 1978. At that meeting, hosted by Dr. John Torrey, an icon in the field of Frankia research, the common interests of a small group of researchers had evolved from developments in three areas. The first area of advancement was the effort, sponsored by the International Biological Program, to establish a worldwide accounting for actinorhizal plants, then known to be comprised of 15 genera in seven "diverse" families. These morphologically "diverse" groups are now known to cluster together with other plant taxa, including legumes, in the subclade rosidae, apparently a taxonomic unit with plants predisposed to diazotrophic symbioses. The second impetus came from the increased recognition by foresters and other natural resource managers that actinorhizal plants possessed nitrogen fixation capacities equal to those of legumes and that actinorhizal plants conferred the dual benefits of superior host-plant productivity on poor soils and enhancement of soil nitrogen fertility. The third development was the first successful isolation of a Frankia strain (CpI1 from Comptonia peregrina) in pure culture and reinfection of a host plant by this isolate. This feat opened the door to a detailed understanding of this nitrogen-fixing actinomycete, its symbiosis with angiospermous host plants, and its potential for genetic improvement through biotechnology.

Since the first conference, and even the most recent one held at the University of Umeå in Sweden in 2006, substantial changes in emphasis and developments have occurred and were featured at this 15th meeting in Argentina. These included (1) notable advancements in 
functional genomics and proteomics of Frankia, (2) the exploration of diversity of Frankia and non-Frankia actinomycetes in nodules, (3) genetic transformation of host plants, (4) understanding regulation of infection and nodulation, (5) the expansion of ecological and applied studies to new locales worldwide, and (6) evaluating the contribution of actinorhizal plants to increased soil $\mathrm{N}$ fertility. It has become apparent that parallels between the leguminous and actinorhizal systems, and even within the groups of actinorhizal plants themselves, may not exist uniformly for such basic, co-evolved processes as recognition and infection of the symbiotic partners.

In spite of the importance of actinorhizal plants to the nitrogen economies of ecosystems worldwide, research and research funding on this symbiotic partnership have been limited. This is likely due to the problems of isolating and manipulating slow-growing Frankia in culture, the difficulty in developing transformed partners for genetic studies, and the fact that host plants are virtually all trees or shrubs of forests, wetlands, grasslands and arid lands, and not primary agricultural or horticultural crops.

Frankia was first isolated in pure culture in 1978, despite decades of effort and, even today, no isolate has been obtained for the major Frankia group responsible for the nodulation of rosaceous and some other host plant taxa. Because there are a limited number of researchers focusing on Frankia and actinorhizal plants, progress lags behind that achieved for the nitrogen-fixing symbioses of leguminous plants. Nonetheless, actinorhizal symbioses are of fundamental importance as a unique symbiotic system in nature. They play a major role in maintaining the function and biotic diversity of a variety of extensive ecosystems worldwide. At the same time actinorhizal plants are playing an increasingly prominent role in plantations. Indeed, hardy, exotic casuarinas are today the most important tree species grown in Egypt and are widely planted in tropical and subtropical regions throughout the world. As we learn more about actinorhizal plants, their usefulness will continue to expand in forestry, range management, wildlife habitat management, mine spoil reclamation, soil stabilization, windbreaks, stream bank stabilization, horticulture and agroforestry. For all the reasons mentioned above, we hope that this issue of Symbiosis will encourage more, dedicated researchers to actively pursue research on the actinorhizal symbiosis and attend the next conference which is tentatively scheduled to take place in Portugal in 2010.

We express our thanks to all participants for their contributions to this conference and to the institutions that supported the meeting: Universidad Nacional de Quilmes; Universidad Nacional del Comahue; CONICET, Agencia Nacional de Promoción Científica y Tecnológica; Parque Nacional Nahuel Huapi; and Ministerio de Ciencia, Tecnología e Innovación Tecnológica. We also are grateful for support from the Institut de Recherche pour le Développment (France). Thanks are extended to the editorial staff of Symbiosis, especially to David Richardson and Suzanne Trauffer, for their help in publication of these papers.

This issue is dedicated to the memory of Yvon R. Dommergues, a pioneer in, and major contributor to, actinorhizal biology, particularly advancements in the knowledge of Casuarina symbiosis.

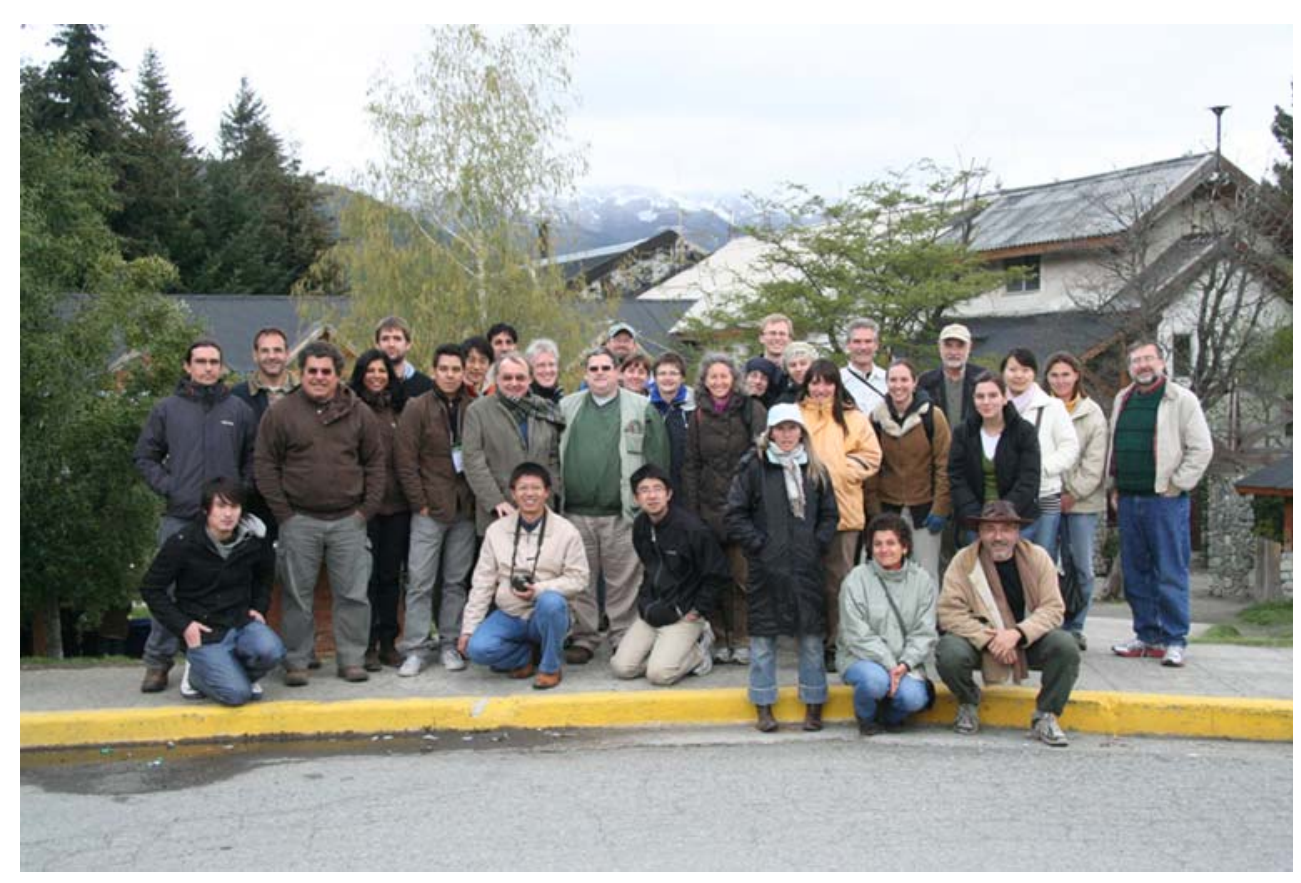

